\title{
CAA e 4Co: opportunità didattiche della comunicazione aumentativa e alternativa su dispositivi mobili
}

\section{Marco Lazzari}

Università di Bergamo, Dipartimento di Scienze Umane e Sociali

doi: 10.7358/ecps-2014-010-lazz

marco.lazzari@unibg.it

\section{AAC AND 4CO: EDUCATIONAL OPPORTUNITIES OF THE AUGMENTATIVE AND ALTERNATIVE COMMUNICATION ON MOBILE DEVICES}

\section{Abstract}

In this paper we present an experience of the application of Augmentative and Alternative Communication $(A A C)$ with four persons with severe language and motor impairments. Two applications (apps) on Android tablets were tried with one subject who had negatively experienced traditional communication boards at school and with three subjects who had never been considered before for AAC intervention. After a six-month trial, based on the co-construction of a personalized communication table with each of the four subjects, we achieved encouraging results: the ease of use of the proposed instruments enabled the persons involved in the experimentation to improve their communication capabilities, to better express their choices and feelings, and to extend their communication circles. These results show that $A A C$ can be successfully applied to severe disabilities and encourages research in this direction. The experience, which is in progress in other contexts and with other pathologies, was heavily time-consuming with reference to the efforts required by the personalized development phase. For the future, we propose a mix of universal design and personalization so that the resulting tools can be regarded as convenient, competent, consonant and contextual.

Keywords: Augmentative and alternative communication, Co-construction, Language impairments, Mobile computing, Social inclusion. 


\section{INTRODUZIONE}

Nel giro di una quarantina d'anni in Italia l'approccio educativo nei confronti delle persone con disabilità è radicalmente mutato. Nel sistema scolastico ciò ha indotto una evoluzione dall'antico sistema esclusivo dell'istruzione separata e delle scuole differenziali e speciali, passata attraverso le fasi dell'inserimento e dell'integrazione (Gelati, 2004; D'Alonzo, 2008), fino ad arrivare all'attuale scuola inclusiva (Pavone, 2010: Medeghini \& Fornasa, 2011), che mira a garantire il diritto soggettivo di tutti all'istruzione, così come previsto dalla Costituzione e chiarito e riaffermato con forza dalla sentenza della Corte Costituzionale n. 215 del 3 giugno 1987. Ne consegue che la scuola dei nostri giorni si prende cura di molteplici forme di disabilità fisica e psichica, dei disturbi specifici dell'apprendimento e dei bisogni educativi speciali e in ambito pedagogico si riflette addirittura sulla necessità di un approccio che conduca a riconsiderare il bisogno, superandone la dimensione passiva e rigida a favore del desiderio, attivo e flessibile (Sandrone, 2012).

$\grave{E}$ da rilevare che il nostro Paese è da sempre all'avanguardia in questo settore (Meijer, Soriano, \& Watkins, 2004; De Anna, 2007; Lascioli, 2007) e che in numerosi altri Paesi il diritto all'istruzione delle persone con disabilità è tuttora un diritto condizionato (Troilo, 2012). In Gran Bretagna, per esempio, una recente sentenza della Corte Suprema, sollecitata da un ricorso della famiglia di un giovane affetto da una grave forma di disturbo dello spettro autistico, ha evidenziato come dalla vigente normativa non discenda per la pubblica amministrazione l'obbligo di fornire prestazioni di inserimento scolastico a persone con bisogni speciali e che dunque l'amministrazione stessa può rifiutare le richieste delle famiglie qualora le sue risorse non siano adeguate a farvi fronte (Osti, 2011).

Pur nella consapevolezza che il nostro Paese gode di un simile "primato inclusivo", è noto che in alcuni casi di disabilità grave i percorsi di scolarizzazione non approdano a molto più di ciò che il Decreto del Presidente della Repubblica n. 104 del 12 febbraio 1985 e la successiva Circolare Ministeriale n. 262 del 22 settembre 1988 del Ministero della Pubblica Istruzione deprecavano come «semplice socializzazione in presenza». Ne sono esempio i quattro casi con i quali è partito il nostro esperimento d'uso di tecnologie informatiche mobili per lo sviluppo di competenze comunicative: si tratta, infatti, di quattro persone con disabilità particolarmente gravi, passate per percorsi di istruzione formale che non hanno lasciato in loro tracce particolarmente evidenti, come sarà chiarito nel seguito. 


\section{INFORMATICA E DISABILITÀ}

L'uso di strumenti informatici nei processi di apprendimento formali, non formali e informali può essere a buon diritto considerato un'efficace risposta tecnologica alla richiesta di ricerca e sviluppo promossa dalla Convenzione delle Nazioni Unite sui diritti delle persone con disabilità (ONU, 2006), ratificata dal Parlamento italiano con la Legge n. 18 del 3 marzo 2009. La Convenzione, infatti, impegna gli Stati Parti a «intraprendere o promuovere la ricerca e lo sviluppo, ed a promuovere la disponibilità e l'uso di nuove tecnologie, incluse TIC, ausilii alla mobilità, dispositivi e tecnologie di sostegno, adatti alle persone con disabilità, dando priorità alle tecnologie dai costi più accessibili» (art. 4). Nello stesso documento, la sezione intitolata significativamente "Vita indipendente e inclusione nella società" prevede che gli Stati Parti, riconosciuto il diritto di tutte le persone con disabilità a vivere nella società, adottino «misure efficaci ed adeguate al fine di facilitare [...] la loro piena integrazione e partecipazione nella società» (art. 19). Poco oltre gli Stati Parti si impegnano perché vengano adottate «tutte le misure adeguate a garantire che le persone con disabilità possano esercitare il diritto alla libertà di espressione e di opinione, ivi compresa la libertà di richiedere, ricevere e comunicare informazioni e idee su base di uguaglianza con gli altri» (art. 21).

Parimenti, opportuni strumenti informatici possono soddisfare anche le richieste del Communication bill of rights (USA), contenute nelle Guidelines for meeting the communication needs of persons with severe disabilities (NJC National Joint Committee for the Communication Needs of Persons with Severe Disabilities, 1992), che auspicano che alle persone con disabilità gravi sia garantito il diritto di esprimere preferenze personali o sentimenti (art. 1), di poter avere a disposizione scelte e alternative (art. 2), di poter richiedere e dare attenzione e ottenere l'interazione con un'altra persona (art. 4) e di proporsi come agenti comunicativi a tutti gli effetti verso gli altri (art. 9). Lo stesso obiettivo viene indicato in termini analoghi dalla Commissione della Comunità Europea con la sua iniziativa eInclusion, che mira a permettere a ogni persona di partecipare pienamente alla società dell'informazione, indipendentemente da svantaggi individuali o sociali (Commission of the European Communities, 2007).

Le opportunità offerte alle persone con disabilità dall'attuale stato della tecnologia dell'informazione derivano innanzitutto da un processo che ha caratterizzato la storia del calcolatore dai primordi fino ai giorni nostri, quello della miniaturizzazione (Lazzari et al., 2010). Con questo termine ci riferiamo in primo luogo alla continua riduzione delle dimensioni della componentistica elettronica, meccanica e ottica e alla conseguente riduzione delle dimensioni complessive dei sistemi di elaborazione: comuni calcolatori da 
scrivania o portatili del giorno d'oggi sono in grado di fornire prestazioni, in termini di velocità di esecuzione o di capacità di memorizzazione, superiori a quelle di calcolatori che negli anni Cinquanta-Sessanta occupavano interi edifici.

Contemporaneamente si è verificata una corrispondente miniaturizzazione dei costi dei sistemi di elaborazione elettronica, tale per cui la potenza di calcolo che solo trent'anni fa era ottenibile a fronte di una spesa di miliardi di lire è ora facilmente disponibile con spese dell'ordine delle centinaia di euro. Proprio una trentina di anni fa o poco più, fra il 1977 e il 1981, esordivano appunto i capostipiti delle due grandi famiglie di elaboratori personali che si sarebbero poi affermate in tutto il mondo: l'Apple II (precursore della famiglia Macintosh) e il Personal Computer IBM 5150 (che avrebbe dato il nome alle macchine che oggi genericamente chiamiamo PC). La nascita dell'elaborazione personale era stata permessa dal convergente processo di compressione delle dimensioni dei calcolatori e di diminuzione dei loro prezzi, al punto che l'industria informatica era ormai in grado di produrre apparecchiature le cui dimensioni permettevano loro di essere poste su una scrivania senza pregiudizio della sua stabilità (desktop computer) e di essere acquistate da una piccola azienda o da un privato senza pregiudicare il bilancio societario o familiare.

La miniaturizzazione ha concorso a far decollare il mobile computing, che ci offre computer che hanno cessato di essere ciò che erano agli albori della storia dell'informatica - strumenti di calcolo muniti di dispositivi di comunicazione per scambiare risultati con il mondo esterno - e sono piuttosto percepiti dagli utenti come strumenti di comunicazione dotati anche di funzionalità di calcolo per elaborare flussi di comunicazioni da e verso il mondo esterno (Lazzari, 2006).

In altri termini, la miniaturizzazione della componentistica ha generato due principali proprietà dei dispositivi informatici contemporanei: portabilità e trasparenza. Entrambi hanno un grande impatto sui possibili impieghi da parte di persone con disturbi del linguaggio, quali quelli oggetto del nostro lavoro. La portabilità favorisce l'uso in mobilità, in modo che lo stesso dispositivo può essere sfruttato per comunicare in diversi microsistemi ecologici (Bronfenbrenner, 1979) - nel caso della nostra sperimentazione la famiglia e il centro diurno dove si è svolta l'esperienza iniziale - e anche per favorire le interazioni mesosistemiche - per esempio, tra famiglia e centro diurno. Per quanto riguarda la trasparenza, si intende qui la proprietà dei sistemi informatici di proporsi con implementazioni spazialmente discrete, non ingombranti né invasive, che abbassano la soglia psicologica di accettazione dei dispositivi assistivi da parte dei loro potenziali utenti (Lazzari, 2013); si tratta della proprietà per la quale, per esempio, l'alunno dislessico accetta più fa- 
cilmente di usare il tablet, laddove rifiuta il tradizionale PC sul banco, che lo può aiutare, ma che con il suo ingombro evidenzia la condizione «differente» dell'alunno e lo stigmatizza.

Come mostreremo nel seguito, il combinato disposto delle due proprietà fa sì che oggigiorno siano disponibili soluzioni informatiche che non agiscono soltanto da protesi funzionali (il grado zero delle tecnologie assistive, nella loro funzione compensativa di strumenti che permettono l'accesso al computer, come per esempio nel caso di tastiere espanse, puntatori alternativi al mouse o lettori di schermo), ma fungano anche da protesi sociali, configurandosi dunque non solo come amplificatori cognitivi, ma addirittura come amplificatori sociali (Mangiatordi \& Pischetola, 2010), in grado di combattere la perdita di rilevanti opportunità informative, lavorative, educative e di partecipazione sociale e politica per le persone con disabilità (Bentivegna, 2009), ovvero la perdita di significative opportunità esistenziali (van Dijk, 2005).

\section{LA COMUNICAZIONE AUMENTATIVA E ALTERNATIVA}

Comunicazione Aumentativa e Alternativa (CAA) è un termine generico e ampio, usato per indicare molteplici metodi e tecniche che aiutano le persone con difficoltà linguistiche a integrare (aumentativa) o sostituire (alternativa) il linguaggio naturale o la scrittura e a capire o produrre linguaggio parlato o scritto.

Secondo la prospettiva della CAA, dunque, una persona che, per qualsivoglia ragione, non sia in grado di esprimere il proprio pensiero verbalmente, lo può trasmettere con modalità diverse dalla parola (Gava, 2013); e chi non sia in grado di intendere il linguaggio scritto, può essere aiutato con modalità alternative a stampa e scrittura.

La CAA si propone come strumento finalizzato a contenere o eliminare il senso soggettivo di isolamento vissuto dalle persone con disabilità comunicativa grave: essa infatti offre l'opportunità di mettersi in comunicazione con gli altri in maniera efficace per esprimere i propri bisogni, per condividere informazioni, per stabilire e mantenere relazioni e per conformarsi alle convenzioni sociali (Light, 1988); permette anche, in un certo senso, di comunicare con sé stessi, per esempio nel momento in cui consente alle persone di autogestirsi le attività quotidiane mantenendone un'agenda redatta con strumenti alternativi alla scrittura (Beukelman \& Mirenda, 2013).

Gli interventi basati su metodi di CAA si caratterizzano rispetto ad altre tipologie di terapie riabilitative nel campo del linguaggio per due dimensioni di pluralità: sono multimodali, nel senso che generalmente propongono 
diverse modalità comunicative combinate, come nel caso dell'uso di simboli iconici sullo schermo di un computer accompagnati dalla sintesi vocale delle etichette corrispondenti, oppure nel caso del modeling, la strategia di lettura nella quale un partner comunicativo della persona con disabilità legge ad alta voce un libro modificato con simboli, indicando via via con un dito i simboli nel testo (Costantino, 2012; Fumagalli et al., 2012); e sono relazionali, nel senso che, proprio come nel caso del modeling, non sono diretti unicamente al singolo, ma coinvolgono in maniera attiva la sua rete sociale (e sperabilmente consentono di estenderla).

Non esiste una tipologia unica di soggetti destinati ad avvalersi della CAA. Ciò che accomuna le persone che vi possono ricorrere è la necessità di assistenza adattiva negli atti comunicativi, in quanto la loro capacità di espressione o comprensione è temporaneamente o permanentemente inadeguata allo scopo (Beukelman \& Mirenda, 2013). Di conseguenza la CAA è tipicamente adottata per diverse condizioni o patologie congenite, acquisite o temporanee, quali paralisi cerebrale, ritardo mentale, autismo, disprassia verbale, sclerosi laterale amiotrofica, morbo di Parkinson, sclerosi multipla, demenza, afasia e lesioni cerebrali traumatiche.

Nella molteplicità di metodi alla quale si faceva cenno nella definizione di CAA, si è soliti individuare due famiglie:

- comunicazione unaided (senza ausili): raccoglie le strategie per le quali gli eventi comunicativi non richiedono strumenti di supporto, ma solo l'uso del corpo, come nel caso di espressioni facciali, gesti o lingue dei segni; sono metodi dalle grandi potenzialità espressive, ma generalmente richiedono motricità fine;

- comunicazione aided (con ausili): si tratta di tutti i casi nei quali interviene un ausilio tecnologico (non necessariamente informatico: la CAA nasce negli anni Cinquanta, quando i calcolatori erano ancora quegli oggetti monumentali dei quali si discuteva pocanzi).

Con il termine ausilio ci riferiamo, in accordo con lo standard internazionale ISO 9999:2011, a quei dispositivi, apparecchiature, strumenti e software che possono essere usati da o per persone con disabilità per migliorare la partecipazione alla vita quotidiana, per proteggere, sostenere, sviluppare, controllare o sostituire strutture o funzioni corporee, e per prevenire menomazioni, limitazioni nelle attività od ostacoli alla partecipazione. La letteratura suggerisce che un ausilio dovrebbe soddisfare tre requisiti: essere competente, consonante, contestuale (Mainini, Ferrari, \& Zini 1982), ossia svolgere con efficacia il proprio compito, con soddisfazione dell'utente e in maniera adeguata al contesto d'uso.

Le soluzioni di CAA aided possono a loro volta essere classificate in tre categorie, rispettivamente di bassa, media e alta tecnologia. Le soluzioni a 
bassa tecnologia sono quelle fornite da ausili che non necessitano di energia elettrica, come per esempio le cosiddette tabelle di comunicazione, che permettono agli utenti, a seconda delle loro limitazioni fisiche e cognitive, di costruire discorsi selezionando lettere, parole o simboli disegnati su fogli di carta o cartoncino. Sono stati sviluppati a questo proposito diversi sistemi simbolici, che sono sfruttati anche in soluzioni a media e alta tecnologia; tra di essi i più diffusi sono i simboli Bliss (Figura 1) e i Picture Communication Symbols (PCS; Figura 2).

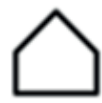

house
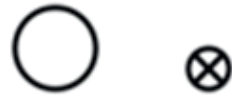

sun
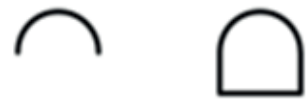

wheel mind knowledge

Figura 1. - Simboli Bliss (CC BY-SA 3.0 by Blissymbolics Communication International, http://www.blissymbolics.org).
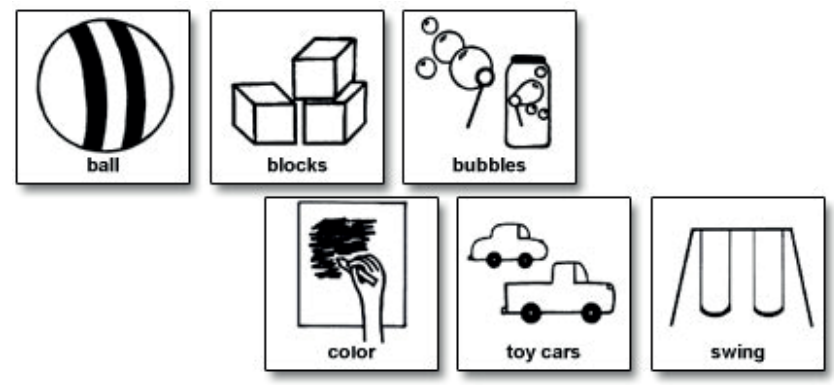

Figura 2. - PCS - Picture communication symbols (CDo2Learn, http://www.do2learn.com).

Le soluzioni a media tecnologia sono quelle fornite da dispositivi elettronici di comunicazione che non richiedono l'uso o la connessione a un calcolatore, come i cosiddetti VOCA (Voice Output Communication Aids; Figura 3). Tipicamente si presentano sotto forma di tabelle di comunicazione composte da vari pulsanti, a ciascuno dei quali è collegato un simbolo o un messaggio preregistrato: alla pressione del pulsante, un sintetizzatore vocale legge la parola o frase che gli corrisponde. Le soluzioni ad alta tecnologia sono quelle che impiegano e integrano calcolatori, interfacce multimediali con acquisizione da fonti esterne (per esempio fotocamera digitale) e sintesi vocale. 


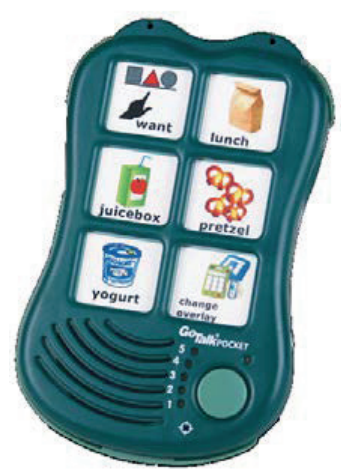

Figura 3. - Un VOCA (Voice Output Communication Aid), il Go Talk Pocket capace di gestire 30 immagini/messaggi

(C) Attainment Company, Inc., http:// www.attainmentcompany.com/).

Questa classica tripartizione fa riferimento all'ambiente di fruizione delle soluzioni CAA; è da tenere presente però che esistono esperienze nelle quali si distingue una fase di produzione, in ambiente informatico, da una di fruizione, che può essere indipendente dal supporto digitale. È tale per esempio il caso degli IN-books (Costantino, 2012), libri su misura pensati per bambini con disabilità comunicative, il cui testo è completamente tradotto in simboli: in questi casi l'editing è tipicamente realizzato su computer (per esempio con programmi come Symwriter) per costruire storie che possono essere poi fruite sia via computer, sia in versione cartacea.

Tra i prodotti ad alta tecnologia i tablet sembrano destinati a diventare strumenti d'elezione per la CAA, poiché già surclassano i dispositivi CAA di prima generazione, basati su PC personalizzati, per quanto riguarda risoluzione dello schermo, densità di pixel, durata della batteria, peso e prezzo (Dolic, Pibernik, \& Bota, 2012), ma anche in considerazione degli aspetti legati all'accettazione psicologica di dispositivi di dimensioni ridotte alla quale si faceva cenno in precedenza.

La letteratura in materia è ancora limitata, ma esistono già confortanti studi circa l'efficacia di ausili basati su $i O S$ (il sistema operativo per dispositivi mobili sviluppato da Apple e disponibile soltanto su apparecchiature Apple) per le persone con disturbi cognitivi e/o dello spettro autistico (Kagoara et al., 2013); l'appropriatezza di apps per persone con bisogni comunicativi complessi (Alliano et al., 2012); le opportunità offerte a studenti con bisogni educativi speciali da soluzioni di mobile learning che consentono loro di svolgere attività che erano impensabili fino a un recente passato (Fernández-López et 
al., 2013); e circa l'effetto positivo (Higginbotham, 2011) che potrebbero avere sulla CAA i dispositivi basati su Android (il sistema operativo per dispositivi mobili, televisioni, console da gioco, fotocamere digitali e altro distribuito da Google e ormai diventato il più diffuso nel mondo della mobilità).

Come vedremo nel seguito, il nostro credo è che la tecnologia è cruciale per affrontare i disturbi del linguaggio, ma ancor di più la capacità di sfruttarla efficientemente (Cook, 2011); ciò richiede, oltre che competenza tecnica, un solido punto di vista metodologico e pedagogico e un approccio centrato sulla persona per adattare il software personalizzabile alle esigenze specifiche e particolari di ogni persona che impieghi soluzioni CAA digitali.

\section{UNA RICERCA-AZIONE SULL'USO DELLA CAA IN CASI DI DISABILITÀ GRAVE}

In questo paragrafo descriveremo sinteticamente un'esperienza d'uso della CAA con disabili gravi, che rappresenta il primo passo di un progetto in corso in diversi ambienti e applicato a disabilità ed età varie.

La prima esperienza è consistita nel tentativo di proporre la CAA su dispositivi mobili a quattro adulti con gravi deficit del linguaggio e del movimento, ospitati presso un centro diurno per persone con disabilità. Ciò è stato fatto attraverso una ricerca-azione partecipativa (McIntyre, 2008), compiuta in un arco di tempo di sei mesi (set. 2012 - feb. 2013), che ha previsto l'uso di due apps in esecuzione su un tablet. I quattro soggetti sono stati supportati da un'assistente, che progressivamente li ha addestrati nell'uso di un tablet e ha co-costruito con loro opportune tabelle comunicative personalizzate per aiutarli nelle interazioni conversazionali di base di due tipi: la scelta delle attività da svolgere presso il centro e quella del loro piatto preferito dal menu del pranzo. Per monitorare le fasi e i risultati dell'attività è stato compilato un diario quotidiano. Di seguito, proponiamo e discutiamo una sintesi delle principali evidenze registrate nel corso dell'esperimento e riviste riflessivamente.

\subsection{Scelta dei candidati}

Nella comunità scientifica non c'è allo stato attuale particolare consenso circa l'esistenza di criteri di candidatura per gli interventi di CAA (Romski \& Sevcik, 2005). Per parte nostra, abbiamo deciso di selezionare per il nostro programma persone che fossero in grado di manifestare chiara intenzionalità di comunicazione e di esprimere in maniera piuttosto comprensibile la differenza tra il sì e il no mediante comunicazione verbale o non verbale (o entrambe). La 
nostra scelta è caduta su quattro persone di età compresa tra i 20 e i 30 anni, una delle quali era già stata esposta senza successo ai metodi tradizionali CAA a scuola, mentre le altre tre non erano mai state neppure prese in considerazione prima per interventi di CAA, in quanto la si riteneva inadeguata per i loro casi. Pur avendo tutti e quattro esperito percorsi di scolarizzazione fino ai sedicidiciotto anni, nessuno di loro aveva acquisito competenze di letto-scrittura.

Il numero di soggetti per la prova è stato limitato a quattro perché era disponibile una sola assistente di ricerca per la sperimentazione e per ragioni legate all'organizzazione delle attività del centro diurno che ospitava la ricerca.

Per rendere l'idea del tipo di situazioni affrontate, sintetizziamo le condizioni dei quattro soggetti coinvolti (nella scrupolosa osservanza degli accordi presi con le famiglie per il rispetto della privacy):

- Tizio, 29 anni: insufficienza mentale grave correlata ad afasia e sospetta sindrome di Angelmann; limitazioni coxofemorali da osteotomia di Chiari.

- Caio, 26 anni: ritardo mentale medio grave, sindrome comiziale pregressa, tetraparesi prevalente agli arti inferiori, epilessia generalizzata non convulsiva.

- Sempronia, 22 anni: sindrome di Down, con grave compromissione dell'area del linguaggio, ipotiroidismo congenito e scoliosi dorso-lombare; nessuna compromissione funzionale a livello motorio, buone abilità finomotorie, ma problemi di vista.

- Gaia, 21 anni: sindrome di Joubert con grave compromissione dello sviluppo cognitivo, assenza del linguaggio espressivo e di autonomie personali; compromissioni motorie di autonomia di spostamento gravi, nonché compromissioni delle abilità fino-motorie.

E evidente che ai disturbi del linguaggio si associano anche problemi motori o di acuità visiva che rendono problematico l'uso di strumenti informatici.

\subsection{Obiettivi}

All'inizio della ricerca-azione abbiamo definito alcuni obiettivi rispetto all'uso del dispositivo da parte dei soggetti coinvolti nel progetto:

- Ob. 1: sviluppare una motivazione all'uso del dispositivo per comunicare.

- Ob. 2: usare correttamente il dispositivo con gli educatori.

- Ob. 3: migliorare la coordinazione oculo-manuale.

- Ob. 4: usare il dispositivo in diversi contesti.

- Ob. 5: usare il dispositivo per manifestare bisogni espressivi primari.

- Ob. 6: creare o aumentare la consapevolezza della partecipazione alla vita quotidiana.

- Ob. 7: aumentare la motivazione a comunicare con altre persone oltre agli educatori del Centro. 


\subsection{Scelta del dispositivo e del sistema operativo}

Per ragioni legate ai costi, abbiamo deciso di optare per una piattaforma hardware non legata a $i O S$, sembrandoci economicamente più ragionevole da proporre alle famiglie per un eventuale futuro acquisto. Si è scelto dunque di puntare sul sistema operativo Android in virtù della sua diffusione, dell'economicità (relativa) di gran parte dei dispositivi che lo montano e del numero di potenziali applicazioni gratuite disponibili sul mercato.

Abbiamo svolto alcuni test preliminari su diversi tablet: dopo una prima tornata, abbiamo deciso di scartare gli schermi di dimensioni inferiori ai 10 pollici, in quanto non li abbiamo ritenuti adatti a persone con limitata acuità visiva e/o ridotte capacità motorie quali i soggetti del nostro progetto. Infine, abbiamo scelto un Samsung Galaxy Tab 210.1 (con processore dual Core da $1 \mathrm{GHz}, 1 \mathrm{~GB}$ di RAM, schermo da 10.1" e $1280 \times 800$ px) con sistema operativo Android versione 4.0.4 Ice Cream Sandwich. Alcuni esperimenti sono stati eseguiti anche su un più piccolo Samsung Galaxy Tab 27 e, dopo la conclusione della sperimentazione al centro diurno, su uno smartphone Google Nexus 5 e su un tablet ASUS Transformer Pad TF300TL.

\subsection{Scelta del software applicativo}

Per quanto riguarda il software, sono state scaricate varie apps dalla piattaforma Google Play, che sono state provate e valutate alla luce delle esigenze di progetto, vale a dire l'obbligo di disporre di:

- licenza gratuita, per evitare vincoli economici per future eventuali adozioni da parte delle famiglie o del centro;

- elevata personalizzazione, per fornire a ciascun soggetto un ambiente adeguato alle sue specifiche necessità;

- facilità d'uso, struttura dell'interfaccia semplice e chiara, per facilitare sia la fase di sviluppo, sia quella di uso vero e proprio;

- interfaccia in italiano o in inglese, per non complicare troppo il compito di sviluppo (nel caso specifico, i soggetti coinvolti non sanno leggere e dunque per loro la questione sarebbe stata irrilevante);

- integrazione di sintesi vocale, per avere un feedback immediato alla pressione dei simboli e per poter ricostruire frasi al termine di vari passaggi concatenati.

Sono state verificate rispetto ai requisiti e scartate una decina di applicazioni (Grigis \& Lazzari, 2013) e alla fine si è deciso di usarne due: JABtalk e AAC Talking Tabs, che permettono entrambe la gestione di categorie di simboli o immagini, l'inserimento di immagini e la generazione di output vocale. La prima prevede una struttura ad albero per organizzare i simboli 
e le immagini che è navigabile con semplicità passo per passo dall'utente; la seconda consente di costruire intere frasi man mano che ci si sposta da un'immagine (o simbolo) all'altra, che possono essere rilette dal sintetizzatore vocale alla fine del processo di costruzione della frase.

\subsection{Co-costruzione con gli utenti di una tabella di comunicazione}

Sin dall'inizio della sperimentazione è stato chiaro che motivare all'uso dello strumento le quattro persone scelte per il progetto sarebbe stato piuttosto difficile, dal momento che, ciascuno con le proprie specificità, si tratta di caratteri spesso oppositivi e ostili verso le figure di autorità e dunque sarebbe stato difficile imporre loro uno strumento di comunicazione già pronto $\mathrm{e}$ calato dall'alto.

Per questo motivo si è scelto di impostare un piano di lavoro che ha previsto che per ogni utente e con il suo coinvolgimento diretto si co-costruisse una tabella di comunicazione personalizzata, sia per quanto riguarda il vocabolario (a ciascuno le proprie immagini), sia per quanto riguarda le caratteristiche delle immagini (per esempio, dimensioni diverse in funzione della capacità di indirizzamento più o meno fine dei soggetti tramite tocco dello schermo). Si è difatti immaginato che soltanto un progressivo coinvolgimento a partire dalla quotidianità ripresa per via fotografica avrebbe indotto $\mathrm{i}$ soggetti al successivo uso del tablet per la comunicazione.

Questa decisione, che a conti fatti si è rivelata vincente, ha però richiesto un processo di sviluppo lungo e faticoso: inizialmente si è dovuto far familiarizzare gli utenti con il dispositivo e le modalità di interazione; poi li si è coinvolti nella ripresa di fotografie che sono state scattate per ogni situazione, oggetto o persona che doveva entrare a far parte del vocabolario del singolo utente (si è fatto uso anche dei simboli PCS, che sono stati preferiti nei casi in cui li si è ritenuti più efficaci); infine, gli utenti sono stati istruiti su come usare il tablet per rispondere alle richieste ed esprimere scelte, desideri o sentimenti.

Il compito di co-costruzione è stato impegnativo anche perché le persone coinvolte soffrono di mancanza di concentrazione o hanno una capacità di attenzione limitata e le fasi di sviluppo sono dovute essere strategicamente precedute e intercalate da momenti di intrattenimento e rinforzo.

Un rinforzo utile per il processo è venuto dal fatto che i soggetti hanno apprezzato sin da subito l'uso del tablet per i momenti di svago e ciò li ha fidelizzati all'uso del dispositivo e ha creato un'atmosfera di consenso che ha facilitato la positiva evoluzione della situazione. 


\section{Risultati}

Al termine della fase di sviluppo e di formazione, tutti e quattro gli utenti hanno mostrato interesse per la nuova forma di comunicazione (Ob. 1 del par. 4.2), che hanno imparato ad adoperare in modo proprio (Ob. 2 e Ob. 3), richiedendo di ricorrervi senza bisogno di stimoli dagli educatori. Hanno acquisito capacità di comunicazione in situazioni diverse (Ob. 4), ciò che ha permesso loro di esprimere le loro preferenze, per esempio per la scelta di cosa mangiare o bere (Ob. 5): per fare un esempio, Tizio ora sceglie sistematicamente caffe espresso dopo pranzo, mentre per più di un anno gli era stato servito regolarmente caffe d'orzo, perché il personale era convinto che lo preferisse all'espresso. Hanno sfruttato la possibilità di creare eventi comunicativi che vanno al di là della struttura conversazionale domanda-risposta che ha sempre costituito la loro unica modalità espressiva; vale ricordare che, per le loro competenze linguistiche, la risposta si limita a sì/no, configurando gli eventi comunicativi come alberi binari, i cui nodi corrispondono ciascuno a una domanda e i cui archi sono etichettati con le risposte sì e no rispettivamente. A titolo di esempio, tra Sempronia e l'educatrice ha avuto luogo la seguente discussione (Figura 4): «Posso avere un altro biscotto? No [Sempronia era a dieta]. Allora io li mangio a casa» (Ob. 5 e Ob. 6). Ė evidente quanto un tipo di interazione del genere sia difficilmente esprimibile in uno schema eterodiretto di domande e risposte sì/no.

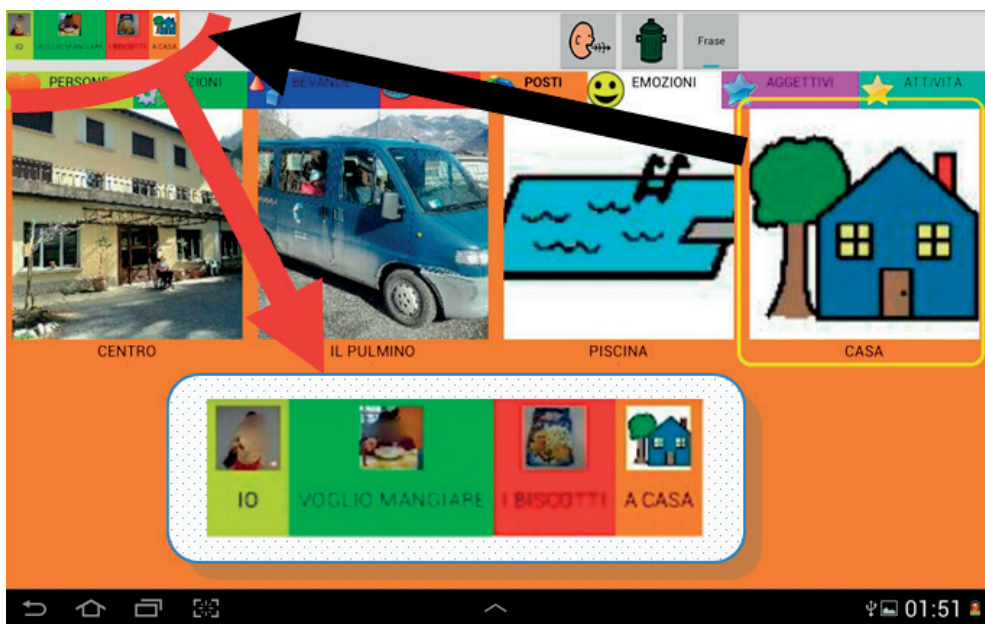

Figura 4. - Quarto passo della costruzione della frase: "Io voglio mangiare i biscotti a casa" con AAC Talking Tabs (immagine rielaborata, per evidenziare la costruzione della frase che avviene nel margine superiore sinistro dello schermo). 


\section{Discussione}

Lobiettivo principale della nostra ricerca-azione esplorativa è stato verificare se i dispositivi tablet (e le relative apps) fossero appropriati per facilitare la comunicazione di persone con disabilità gravi come quelle coinvolte nell'esperienza. I risultati del lavoro sono più che soddisfacenti e ci hanno indotto a dar seguito all'esperienza, che sta continuando con soggetti che hanno patologie ed età diverse.

Con riferimento alla scelta del tablet, questo dispositivo si è rivelato facile e intuitivo da usare anche da persone che sono fisicamente e cognitivamente molto compromesse, data l'immediatezza e fluidità dell'approccio di interazione tramite touch-screen. Dalle note del diario delle attività si ricava che i soggetti coinvolti nella ricerca, nonostante le gravi difficoltà motorie (o, in un caso, visive), sono riusciti a interagire facilmente con il dispositivo sin dai primi tentativi grazie alla semplice imitazione dei gesti dell'operatore; l'interfaccia gestita tramite il tocco è stata assimilata secondo le diverse abilità di ciascuno.

La facilità di utilizzo del dispositivo interattivo ha permesso a tutti e quattro i soggetti di estendere le proprie opportunità di comunicazione. Con riferimento ai principi del già citato Bill of rights (NJC, 1992), è stata data concretamente la possibilità di vivere al meglio il loro diritto di «richiedere oggetti azioni, eventi e persone, e di esprimere preferenze personali o sentimenti», e più in generale di poter scegliere tra più alternative (art. 2).

La funzionalità di sintesi vocale è stata fondamentale per aiutarli a capire e ricordare il significato di immagini e simboli cliccati (si rammenta che i soggetti non sono in grado di leggere le scritte che si vedono sotto a ogni immagine nella Figura 4), consentendo loro una verifica immediata dei messaggi costruiti con il tablet. Allo stesso tempo, la sintesi vocale ha permesso di stabilire relazioni di comunicazione con persone diverse, senza la necessità di spiegare il metodo, in modo più trasparente ed efficace di quanto non avvenga quando si ricorra a tabelle CAA ordinarie. Con riferimento al Bill of rights, si implementa così il diritto di interagire con un'altra persona, di chiedere e dare attenzione, e di partecipare a pieno titolo come agenti comunicativi con altre persone (artt. 4 e 9).

La personalizzabilità dei programmi scelti per la sperimentazione e la flessibilità della loro interfaccia grafica sono state di capitale importanza per ottimizzarne l'uso con soggetti diversi e per adattarli alle esigenze di ciascuno.

Il simboli PCS, quando usati al posto delle immagini autoprodotte, si sono dimostrati facili da capire e comunicativamente efficaci. Gli utenti hanno colto intuitivamente il significato della maggior parte dei simboli concreti, mentre per i più astratti una semplice spiegazione dell'educatrice 
era sufficiente per facilitarne la comprensione e l'uso. Le fotografie personali usate come alternative ai simboli sono risultate efficaci per esprimere concetti specifici o comunicare laddove un soggetto sembrava in grado di capire meglio le immagini piuttosto che i PCS.

Infine, si è riscontrato un aumento rilevante dell'intenzionalità della comunicazione, in termini di frequenza di eventi comunicativi promossi autonomamente, che è stata incoraggiata dall'efficacia del sistema e dall'amichevolezza dell'interfaccia. Con riferimento ai dettami del Bill of rights, ciò ha consentito ai soggetti di esprimere preferenze personali o sentimenti (art. 1).

Per riassumere, i nostri risultati mostrano come anche persone con gravi e gravissime compromissioni cognitive e motorie, che spesso sono escluse dalle soluzioni CAA tradizionali, possono comunicare con prodotti che siano adeguatamente personalizzati. Ciò richiede un'intensa (e onerosa) attività di personalizzazione e può essere considerato come una forma inclusiva di cocostruzione centrata sulla persona, volta a riconoscere e valorizzare la dignità e unicità di chi è coinvolto nel progetto di comunicazione, coerentemente con l'impegno costituzionale della rimozione degli «ostacoli [...] che impediscono il pieno sviluppo della persona» (art. 3).

Il fatto che il successo sia arriso a una sperimentazione svolta con persone affette da disabilità così gravi e che non avevano raggiunto obiettivi minimi nei processi di scolarizzazione lascia ben sperare per quanto si potrà fare riproponendo l'esperienza con casi meno gravi ed eventualmente ricontestualizzati in ambienti di apprendimento formali supportati da CAA. È lecito attendersi che casi meno gravi possano muoversi con più autonomia nel costruire le tabelle, ottenendone uno stimolo motivazionale aggiuntivo, tale da favorirne lo sviluppo di competenze comunicative in generale e il successo scolastico in particolare. La sperimentazione sta appunto continuando nelle scuole (infanzia, primaria, secondaria di primo grado).

I due limiti principali dell'esperienza sono legati alle ridotte dimensioni del campione di utenti coinvolti e all'eccessiva mole di lavoro richiesta per le singole personalizzazioni.

Rispetto al primo problema, come già detto il gruppo di lavoro è all'opera per ripetere la sperimentazione con nuovi soggetti e in contesti differenziati.

Per quanto riguarda la questione dei tempi di sviluppo, esaurita la prima fase esplorativa e del tutto votata alla personalizzazione, si impongono due considerazioni.

Da un lato, è evidente che ulteriori sviluppi delle tabelle per le persone già interessate dalla prima fase del lavoro non richiederebbero gli stessi sforzi, in quanto il coinvolgimento dei soggetti è ormai stato ottenuto, così come il loro adattamento alla tecnologia proposta e al tipo di comunicazione. 
D'altro lato, si è compreso che una parte dei vocabolari dei vari soggetti può essere messa a fattor comune (per esempio, non è necessario fotografare con ciascuno dei soggetti la scatola del caffè, una volta che ogni soggetto sia adeguatamente entrato nello spirito del progetto) e si sta lavorando a un archivio di immagini da condividere in stile Wiki.

Ciò andrebbe nella direzione auspicata dall'approccio della Progettazione Universale, intesa in questo contesto come lo sforzo sistematico e proattivo di sviluppare applicazioni informatiche accessibili e usabili da tutti, senza bisogno di soluzioni adattive (Story, Mueller, \& Mace, 1998).

La perentorietà della definizione classica di Progettazione Universale deve essere qui necessariamente mitigata da una proporzione di sforzi di personalizzazione, che compensino opportunamente le perduranti manchevolezze della tecnologia nel suo stato attuale rispetto alla possibilità di adattarsi a tutti i casi. Si tratta di un approccio che mira in prima battuta a rispondere alla maggior parte dei bisogni dei più, senza escludere a priori di ricorrere a un accomodamento ragionevole quando lo si ritenga opportuno per casi specifici (Edyburn, 2005).

La combinazione di una base di Progettazione Universale con una messa a punto personalizzata potrebbe generare soluzioni che, oltre a soddisfare gli anzidetti tre requisiti classici per un ausilio (competenza, consonanza, contestualità), rispettino anche un criterio aggiuntivo di convenienza economica ed ecologica: le soluzioni proposte in questo lavoro si appoggiano ad hardware dai costi contenuti, vi installano software di base e applicativo gratuito, richiederanno in prospettiva uno sforzo di sviluppo, adattamento e addestramento tale da non indurre, circa la ragionevolezza del loro impiego, dubbi del tipo di quelli affrontati dalla Corte Suprema britannica di cui si è riferito in precedenza.

Le soluzioni di CAA qui proposte soddisfano dunque le 4Co del modello di ausilio che ci sentiamo di proporre. Esse sono:

- competenti (o efficienti), poiché raggiungono l'obiettivo prefissato (con il tablet il soggetto aumenta le proprie competenze comunicative);

- consonanti (o confortevoli), poiché gli utenti si trovano a proprio agio nel corso dell'interazione;

- contestuali, poiché si adattano ai vari contesti comunicativi (la portabilità del tablet fa sì che l'uso delle applicazioni non sia confinato sul tavolo da lavoro, come accadrebbe nel caso si usasse un desktop computer);

- convenienti, poiché l'investimento economico iniziale e quello in tempo e risorse cognitive per lo sviluppo e l'addestramento sono compatibili con il contesto d'uso. 


\section{RINGRAZIAMENTI}

Un grazie di cuore a Daniela Grigis, senza la cui passione e perseveranza non sarebbe stato possibile arrivare da nessuna parte. Grazie alle quattro persone con cui abbiamo lavorato, per l'esperienza straordinariamente arricchente che ci hanno fatto vivere e condividere con loro. Grazie al Centro Diurno di Piario (BG) che ha ospitato la fase iniziale del nostro progetto e all'Istituto comprensivo di Borgo di Terzo (BG) che lo sta continuando. Grazie ad Alessandra De Fiori, che con il suo solito entusiasmo ha permesso di continuare la sperimentazione e a Simona Cadei, che ha arricchito gli spunti di riflessione con il suo lavoro svolto su un nuovo filone di applicazione. Grazie a Federica Baroni per la sua consueta disponibilità al confronto.

\section{RIFERIMENTI BIBLIOGRAFICI}

Alliano, A., Herriger, K., Koutsoftas, A. D., \& Bartolotta, T. E. (2012). A review of $21 \mathrm{iPad}$ applications for augmentative and alternative communication purposes. Perspectives on Augmentative and Alternative Communication, 21(2), 60-71.

Bentivegna, S. (2009). Disuguaglianze digitali. Roma - Bari: Laterza.

Beukelman, D., \& Mirenda, P. (2013). Augmentative and alternative communication: Supporting children and adults with complex communication needs (4th ed.). Baltimore, MD: Paul H. Brookes.

Bronfenbrenner, U. (1979). The ecology of human development. Cambridge, MA: Harvard University Press.

Commission of the European Communities (2007). European i2010 initiative on e-Inclusion. http://ec.europa.eu/information_society/activities/einclusion/docs/ i2010_initiative/comm_native_com_2007_0694_f_en_acte.pdf.

Cook, A. (2011). It's not about the technology, or is it? Realizing AAC through hard and soft technologies. Perspectives on Augmentative and Alternative Communication, 20(2), 64-68.

Costantino, M. A. (2012). Costruire libri e storie con la CAA. Gli IN-book per l'intervento precoce e l'inclusione. Trento: Erickson.

D’Alonzo, L. (2008). Integrazione del disabile. Radici e prospettive educative. Brescia: La Scuola.

De Anna, L. (2007). Le politiche di inclusione in Europa e in Italia, dalla scuola di base all'università. In A. Canevaro (a cura di), Lintegrazione scolastica degli alunni con disabilità (pp. 75-84). Trento: Erickson. 
Dolic, J., Pibernik, J., \& Bota, J. (2012). Evaluation of mainstream tablet devices for symbol based AAC communication. Paper presented at KES-AMSTA 2012 Conference, Dubrovnik, Croatia.

Edyburn, D. (2005). Universal design for learning. Special Education Technology Practice, 7(5), 16-22.

Fernández-López, Á., Rodríguez-Fórtiz, M. J., Rodríguez-Almendros, M. L., \& Martínez-Segura, M. J. (2013). Mobile learning technology based on iOS devices to support students with special education needs. Computers \& Education, 61, 77-90.

Fumagalli, L., Reicher, E., Tatavitto, P., \& Junglelink (2012). Storie con la CAA 1. Trento: Erickson.

Gava, M. L. (2013). La comunicazione aumentativa alternativa tra pensiero e parola ( $2^{a}$ ed.). Milano: FrancoAngeli.

Gelati, M. (2004). Pedagogia speciale e integrazione. Roma: Carocci.

Grigis, D., \& Lazzari, M. (2013). Augmentative and alternative communication on tablet to help persons with severe disabilities. Paper presented at CHItaly '13 - Biannual Conference of the Italian Chapter of the ACM SIGCHI, Trento, Italy.

Higginbotham, J. (2011). The future of the Android operating system for augmentative and alternative communication. Perspectives on Augmentative and Alternative Communication, 20(2), 52-56.

Kagoara, D. M., van der Meer, L., Ramdoss, S., O’Reilly, M. F., Lancioni, G. E., Davis, T. N. (2013). Using iPods' and iPads' in teaching programs for individuals with developmental disabilities: A systematic review. Research in Developmental Disabilities, 34(2), 147-156.

Lascioli, A. (a cura di). (2007). Pedagogia speciale in Europa. Problematiche e stato della ricerca. Milano: FrancoAngeli.

Lazzari, M. (2006). Le frecce di Basilea e le faretre degli informatici. In G. Bertagna (a cura di), Scienze della persona: perché? (pp. 219-238). Soveria Mannelli (CZ): Rubbettino.

Lazzari, M. (2013). Di come la miniaturizzazione possa giovare all'insegnamento musicale nei casi di disturbi specifici di apprendimento. In C. Farinella, Musica a scuola - Disturbi specifici dell'apprendimento (DSA) (pp. 9-16). Modena: Artestampa.

Lazzari, M., Bianchi, A., Cadei, M., Chesi, C., \& Maffei, S. (2010). Informatica umanistica. Milano: McGraw-Hill.

Light, J. (1988). Interaction involving individuals using augmentative and alternative communication systems: State of the art and future directions. Augmentative and Alternative Communication, 4, 66-82.

Mainini, M. L., Ferrari, A., \& Zini, M. T. (1982). La nascita: relazione madre, padre, bambino. Atti della Conferenza USL sui servizi materni infantili, Parma, Italy. 
Mangiatordi, A., \& Pischetola, M. (2010). Sustainable innovation strategies in education: OLPC case studies in Ethiopia and Uruguay. Paper presented at the Third World Summit on the knowledge society, Corfu, Greece.

McIntyre, A. (2008). Participatory action research. Thousand Oaks, CA: Sage.

Medeghini, R., \& Fornasa, W. (a cura di). (2011). L'educazione inclusiva. Milano: FrancoAngeli.

Meijer, C., Soriano, V., \& Watkins, A. (2004). L'integrazione dei disabili in Europa. I Quaderni di Eurydice, 23, 1-96.

NJC - National Joint Committee for the Communication Needs of Persons with Severe Disabilities (1992). Guidelines for meeting the communication needs of persons with severe disabilities. http://www.asha.org/docs/html/GL199200201.html.

ONU (2006). Convenzione delle Nazioni Unite sui diritti delle persone con disabilità. New York: ONU.

Osti, A. (2011). Il caso A (appellant) v. Essex County Council (respondent) di fronte alla Corte Suprema britannica: il diritto all'istruzione di soggetti con disabilità è un diritto assoluto? Spunti per una riflessione a più ampio spettro. Rivista AIC (Associazione Italiana dei Costituzionalisti), 1, 1-5.

Pavone, M. (2010). Dall'esclusione all'inclusione: lo sguardo della pedagogia speciale. Milano: Mondadori Università.

Romski, M. A., \& Sevcik, R. (2005). Augmentative communication and early intervention: Myths and realities. Infants \& Young Children, 18(3), 174-185.

Sandrone, G. (2012). Pedagogia speciale e personalizzazione. Brescia: La Scuola.

Story, M. F., Mueller, J. L., \& Mace, R. L. (1998). The universal design file: Designing for people of all ages and abilities. Raleigh, NC: NC State University, The Center for Universal Design.

Troilo, S. (2012). Tutti per uno o uno per tutti? Il diritto all'struzione e all'integrazione scolastica dei disabili nella crisi dello stato sociale. Milano: Giuffrè.

van Dijk, J. A. G. M. (2009). The deepening divide. Thousand Oaks, CA: Sage.

\section{RiassunTO}

Presentiamo un'esperienza d'uso della Comunicazione Aumentativa e Alternativa (CAA) proposta a quattro persone con gravi disabilità linguistiche e motorie. Due applicazioni su tablet Android sono state testate con un soggetto che aveva sperimentato negativamente tabelle di comunicazione tradizionali a scuola e con tre soggetti che non erano mai stati considerati candidabili a interventi con CAA. Uno studio di sei mesi, basato sulla cocostruzione personalizzata di tabelle di comunicazione con ognuno dei quattro soggetti, ha dato risultati incoraggianti: la facilità d'uso degli strumenti ha consentito alle persone 
coinvolte nella sperimentazione di migliorare le capacità comunicative, esprimere meglio scelte e sensazioni ed estendere le proprie reti di comunicazione. I risultati testimoniano che la CAA può applicarsi con successo a disabilità gravi e incoraggia la ricerca anche negli ambienti educativi formali, dove le persone coinvolte nell'esperimento non avevano maturato significative competenze comunicative. L'esperienza, che continua con altri soggetti e altre patologie, si è rivelata onerosa dal punto di vista del tempo e degli sforzi richiesti per lo sviluppo. Per il futuro si prefigura una opportuna combinazione di Progettazione Universale e fasi di personalizzazione, per rendere gli ausili sviluppati anche convenienti, oltre che competenti, consonanti e contestuali.

Parole chiave: Co-costruzione, Comunicazione aumentativa e alternativa, Dispositivi mobili, Disturbi del linguaggio, Inclusione sociale. 